\title{
Indicadores para la detección de maltrato en niños
}

Emilio Herrera-Basto, M.C., M. en Admón. Pub.(1)

$E^{1}$ 1 maltrato a los niños es un fenómeno universal que no tiene límites culturales, sociales, ideológicos ni geográficos; no existe país ni comunidad que escape a él, y se presenta tanto en países industrializados como en aquellos en vías de desarrollo.

Los modelos o métodos de educación de los hijos han cambiado de acuerdo con la época y la cultura, al igual que las características de la familia; sin embargo, el maltrato a los hijos ha persistido.

En épocas pasadas el padre era considerado como el dueño y no como el protector de su hijo -que sería lo más adecuado-, ya que ejercía un poder absoluto sobre él. Podemos citar el derecho paternal que Rómulo otorgó en común a patricios y plebeyos, el cual permitía que los padres pusieran a sus hijos en prisión, los golpearan con varas, los vendieran como esclavos e, incluso, los mataran. También en la América prehispánica, entre los aztecas y los mayas, la autoridad se depositaba en el varón, quien gozaba del derecho de vida y muerte sobre los hijos.

El infanticidio fue muy común en tiempos bíblicos, y como ejemplo de ello tenemos que Abraham estuvo a punto de sacrificar a su hijo Isaac. Hubo un tiempo en el que los niños cristianos eran azotados cada día de muertos para recordar la masacre emprendida por Herodes. En la antigua Palestina era muy común el sacrificio de los primogénitos en honor a los dioses. Otra forma de infanticidio era el emparedamiento que como en Jericó colocaban a los niños en los cimientos de las murallas para supuestamente fortalecerlas. En la India el infanticidio era practicado en niños que nacían con defectos físicos, y en China, hacia 1870, el infanticidio femenino era permitido como una forma de control prenatal; el cuarto hijo era arrojado a los animales salvajes.

La eugenesia era otro argumento a favor del infanticidio; Séneca, Platón y Aristóteles apoyaban el asesinato de los niños defectuosos. El asesinato de los hijos ilegítimos al nacer era muy común en Alemania y Europa hace 200 años. Los japoneses sacrificaban a sus niños como un método de control de la natalidad, y las mujeres sobrevivían porque las podían casar, vender como prostitutas o sirvientas, o convertirlas en geishas.

En Estados Unidos de América (EUA), durante la época colonial, los menores de edad se cedían por contrato durante años para servir como mano de obra barata. Un problema especial existía en relación con los niños de color, para los cuales no existía ningún tipo de atención. Durante el siglo XIX, los niños indigentes eran vendidos a agricultores o artesanos, para que cubrieran jornadas de trabajo de 16 a 18 horas, a cambio de salarios muy bajos. ${ }^{1-3}$

La sociedad empezó a tomar conciencia de la existencia del problema y de la presencia del maltrato hasta finales del siglo XIX. Como un antecedente de fundamental importancia en el estudio del síndrome del niño maltratado, se encuentra el caso de Mary Ellen, primero en EUA que provocó una intervención legal en 1874. Como no existía una ley ni dependencia

(1) Coordinador de Investigación y A ccidentes, Programa de Atención a la Salud del N iño, Secretaría de Salud, México. 
que tuviese facultades para intervenir, en forma irónica el caso fue llevado a la corte avalado por la Sociedad Protectora de Animales. A raíz de este hecho, surge en EUA, en 1875, la Sociedad de Prevención de la Crueldad en los Niños y, posteriormente, se crean otras organizaciones con esta misma función. ${ }^{4-7}$

Henry Kempe definió inicialmente el síndrome como "el uso de la fuerza física en forma intencional, no accidental, dirigida a herir y lesionar o destruir a un niño, ejercido por parte de su padre o de otra persona responsable del cuidado del menor" ${ }^{7-9}$ La Comisión Nacional de los Derechos Humanos, por su parte, lo define como "toda aquella forma extrema de amenazas, represión, castigos o humillaciones que inflijan un sufrimiento físico, psicológico o moral a un ser humano, y más aún si se trata de un menor."10

La actitud de una sociedad hacia los niños es parte de un contexto complejo de valores que pueden variar de una cultura a otra, aun entre pueblos contiguos.

\section{Indicadores de maltrato}

En la práctica médica diaria la detección de maltrato requiere de la búsqueda activa de indicadores; sin embargo, no es frecuente que éstos sean requeridos como motivo de consulta. Además no existen signos patognomónicos ni excluyentes como elementos para el diagnóstico diferencial respecto a otras causas de violencia. Asimismo, el temor del médico a verse involucrado en un proceso que podría tomar curso legal, dificulta el proceso de diagnóstico.

En el examen físico del niño, es importante buscar, constatar o descartar la presencia de signos sugerentes de maltrato.

\section{Indicadores de maltrato fisico}

- Se pueden observar contusiones, equimosis, eritemas, laceraciones, quemaduras, fracturas, deformidad de la región; signos de intoxicación o envenenamiento, así como de traumatismo craneal con daño visceral; huellas de objetos agresores como cinturones, lazos, zapatos, cadenas y planchas. ${ }^{11,12}$ Estas lesiones, que generalmente son múltiples, no están ubicadas en el rango de lo posible para un menor, bien por su localización poco habitual, por su mayor frecuencia e intensidad o porque se trata de lesiones aparentemente inexplicables o que no corresponden a las explicaciones dadas por los padres o el niño.

- En los casos de maltrato crónico, las lesiones descritas pueden coexistir con cicatrices, deformida- des óseas por fracturas antiguas o con secuelas neurológicas o sensoriales.

\section{Indicadores de abuso sexual}

- Ante toda laceración o daño en el área genital de un niño o niña que no se explique claramente como accidental se debe sospechar de abuso sexual, al igual que cuando se presentan enfermedades de transmisión sexual en un niño o preadolescente.

- Las lesiones más frecuentemente encontradas son: equimosis en la entrepierna y en los labios mayores, laceraciones, sangrado, inflamación, himen perforado (en niñas), dificultad para caminar, rastros de semen y, en etapas más tardías, prurito e infecciones frecuentes, y masturbación.

- Es común que el abuso sexual sostenido ocurra con un miembro de la propia familia y de modo progresivo, por lo que no necesariamente se produce violencia física.

\section{Indicadores de abandono}

- Descuido en la alimentación, en la higiene y la ropa, dermatitis de pañal crónica, signos o cicatrices de accidentes domésticos frecuentes y desnutrición.

- Problemas físicos o necesidades médicas no atendidas (heridas sin curar o infectadas, defectos sensoriales no compensados), o bien, ausencia del control y de los cuidados médicos rutinarios.

- Es importante distinguir el abandono físico, como una forma de maltrato, de la falta de cuidado por la escasez de recursos y las dramáticas condiciones de vida que impone la extrema pobreza. Otro elemento a considerar son las dificultades que las familias en condiciones de aislamiento geográfico y social tienen que enfrentar para acceder a la atención médica.

\section{Indicadores psicológicos y emocionales de maltrato}

La detección de estos indicadores debe realizarse mediante la observación, durante la consulta, de la conducta del niño y del adulto que lo acompaña, así como valorando la calidad de la relación entre ambos; además, se debe hacer una búsqueda sistemática de información acerca de la ocurrencia de maltrato.

La obtención de esta información es compleja, no sólo por el ocultamiento y la negación de los adultos temerosos de un castigo legal o de la censura social, sino también porque el propio niño puede negar el 
abuso por temor, por un sentimiento de lealtad hacia su familia o porque piensa que no le van a creer. El secreto, del que participan también el niño y otros miembros de la familia, permite la manipulación de la dependencia afectiva de los niños por parte del adulto responsable.

La dificultad es aún mayor en los casos de abuso sexual, aunque a menudo es posible obtener información de manera indirecta o de testigos ajenos a la familia.

Algunos indicadores psicológicos o emocionales de maltrato están dados por lo siguiente:

- Es frecuente observar en el niño conductas de temor y retraimiento, o bien, de inquietud excesiva y apego inadecuado al profesional o técnico que presta la atención.

- Las explicaciones del adulto son generalmente vagas, minimizadoras y en abierta contradicción con los hallazgos.

- Puede observarse falta de interacción y contacto, así como una excesiva dependencia a algunas de las conductas del adulto que caracterizan al maltrato emocional: descrédito, ridiculización, descalificación, amenazas, indiferencia, o bien, rechazo explícito o implícito.

- Otros indicadores psicológicos y conductuales observados son: bajo rendimiento escolar, inasistencia a clases o retardos frecuentes, hiperactividad, agresividad, rebeldía, desorganización, o bien, se pueden identificar niños tímidos, poco comunicativos y de apariencia descuidada. ${ }^{11-14}$

Situaciones condicionantes y desencadenantes de maltrato

Cuando existen antecedentes o el médico detecta durante la exploración indicadores que permiten suponer la existencia de maltrato, debe preguntarse sistemáticamente por:

1. Las condiciones predisponentes. Estas se pueden encontrar en los padres, los cuidadores, el propio niño y la familia, y han sido descritas como factores de riesgo. Es importante investigar la existencia de situaciones tales como: si el niño trabaja, si asume responsabilidades desproporcionadas, si es llevado a consulta por adultos distintos al cuidador habitual o si hay cambios frecuentes de cuidadores.

2. Los factores o condiciones desencadenantes del maltrato. Estos pueden manifestarse como crisis generadas por eventos vitales estresantes (separaciones, duelo, encarcelamiento, patologías, des- vinculación sociocultural disruptiva). Asimismo, se pueden presentar cuando la acumulación de circunstancias demandan respuesta de la familia y superan su capacidad de resolver conflictos en forma no violenta (periodos de especial vulnerabilidad, cesantía, pobreza, estrés laboral).

\section{Factores de riesgo}

El nivel o la condición de riesgo, que va desde la mayor vulnerabilidad a la mayor protección, depende de la presencia tanto de factores de riesgo como de los mecanismos y factores protectores que interactúan en cada familia; ello determina la mayor o menor probabilidad de que ocurra maltrato.

Las condiciones de riesgo para la presencia de maltrato pueden originarse en los padres, el embarazo, el niño y las características de la convivencia familiar, incluyendo aspectos sociales y culturales relacionados con la violencia:

1. Condiciones de riesgo en los padres

- Padres maltratados, abandonados o institucionalizados en su infancia.

- Madre adolescente, de bajo nivel de escolaridad, con insuficiente soporte conyugal, familiar y social.

- Padres o personas a cargo del cuidado del niño que padecen patología psiquiátrica, alcoholismo o adicción.

- Padres con baja tolerancia al estrés.

2. Condiciones de riesgo por embarazo

- Embarazo no deseado (antecedente de intención de interrumpir el embarazo, rechazo al embarazo, indiferencia y deficiente autocuidado).

- Embarazo de alto riesgo biológico.

- Depresión posparto.

3. Condiciones de riesgo en el niño

- Recién nacido con malformación o prematurez.

- Alteración de los primeros vínculos.

- Hospitalización precoz prolongada.

- Niño portador de patología crónica.

- Niño con discapacidad física o psíquica.

- Niño con conducta difícil.

- Niño no escolarizado, inasistente o de bajo rendimiento, o fracaso escolar. 
- Niño de la calle.

- Niño de la frontera.

- Maltrato étnico.

4. Condiciones de riesgo en la familia

- Familias con antecedentes de conducta violenta.

- Familias con procesos psiquiátricos.

- Familias en etapa de desintegración.

- Familias con antecedentes de alcoholismo.

- Familias desintegradas.

5. Condiciones de riesgo en el entorno

- Condiciones de vida difíciles, marginación, cesantía, pobreza.

- Aceptación cultural de la violencia.

- Ausencia de redes sociales de apoyo a la comunidad.

Ningún factor de riesgo por sí solo explica la ocurrencia de maltrato; tampoco existe una asociación de factores característica de alguna de las formas de maltrato. Se requiere del estudio de cada caso individual y de la acumulación de varios de estos factores para poder catalogar a un niño con maltrato.

\section{Orientaciones para el diagnóstico}

La Décima Clasificación Internacional de Enfermedades no contiene una categoría específica para el maltrato infantil. Es importante utilizar, en lo posible, las categorías que se relacionan con este problema y, especialmente, integrar en el diagnóstico todos los elementos que puedan orientar el manejo del caso.

Los criterios para la calificación de un caso de violencia o maltrato requieren de una ponderación rigurosa de los antecedentes, indicadores y hallazgos clínicos, así como de considerar el derecho a la privacidad de la familia.

En las primeras consultas no siempre es posible confirmar o tener la certeza de que existe violencia o abuso, por lo que la calificación de "alto riesgo o probable" determina igualmente la necesidad de intervenciones específicas.

Una propuesta para la calificación de un caso como "de alto riesgo o de violencia intrafamiliar o maltrato infantil" son los siguientes:

1. Cuando existen signos de maltrato físico, abandono, maltrato emocional y abuso sexual.

2. Cuando existe asociación con antecedentes o situaciones condicionantes y desencadenantes.
3. Cuando hay información suficiente sobre la ocurrencia de situaciones de maltrato: verbalización de parte del niño, reconocimiento de parte del adulto involucrado, testimonio de otras personas y documento judicial o policial.

El mecanismo probable de la violencia o del maltrato tiene una implicación pronóstica y es determinante para el tipo de intervención.

Categorias de familias que sufren situaciones de violencia o maltrato

1. Aquellas en las que el maltrato surge cuando sus integrantes se ven rebasados por situaciones especialmente estresantes (crisis). Estas crisis pueden tener relación con ciclos de la propia familia o ciclos de la vida de sus miembros (hijos adolescentes, inicio de la escolaridad, nacimiento de niños patológicos, familias numerosas con nacimientos muy seguidos), o bien, con eventos traumáticos inesperados (desastres naturales, pérdida de trabajo, muerte).

En estos casos suelen producirse cambios estructurales que crean dinámicas familiares conducentes al maltrato. Al interior de la familia persiste la autocrítica y hay sentimientos de culpa, aunque los adultos involucrados no puedan, por sí solos, poner fin al ciclo de la violencia. En estos casos es poco frecuente el abuso sexual, y el pronóstico suele ser favorable; la familia colabora con el tratamiento en la medida en que hay intención de cambio, la que, a menudo, surge sólo cuando existe apoyo externo.

2. Aquellas en las que el maltrato o abuso es una conducta crónica, a menudo habitual, mantenida en secreto y con elementos de "explicación" o "justificación" de parte del abusador (por educar, por cariño); ello no permite que la familia se viva a sí misma como abusadora. Este discurso, que es compartido por el niño, tiene elementos de chantaje y restringe el acceso de éste a espacios de socialización alternativos. Es frecuente que estos indicadores correspondan a abuso sexual o emocional.

Con frecuencia este tipo de maltrato está relacionado con cuadros patológicos psiquiátricos de tipo psicopático, de tal modo que puede llegar a ser "una forma de mantener un equilibrio grupal patológico". ${ }^{12}$

El pronóstico de la familia como grupo estable suele ser poco favorable, a pesar de que exista una aparente colaboración del adulto responsable. $\mathrm{Su}$ manejo requiere, generalmente, de un desencadenante que haga público el maltrato; la inter- 
vención, por su parte, provoca una crisis que permite el apoyo terapéutico. Sin embargo, cabe aclarar que en estas familias no siempre es posible realizar una intervención terapéutica, por lo que es importante tomar medidas de protección para el niño.

\section{Ruta crítica}

Ante el diagnóstico de un niño o una niña maltratado/a, el médico particular o institucional deberá recurrir a por lo menos uno de los esquemas institucionales creados para la notificación de los casos de maltrato; uno de los más tradicionales es el del Sistema para el Desarrollo Integral de la Familia (DIF) (figura 1).

De acuerdo con dicho esquema, un médico particular o institucional, un familiar, un vecino o cualquier persona que se percate de que un niño es agredido física o psicológicamente, o bien, de que es víctima de abandono o descuido, puede presentar una denuncia ante el DIF. Cuando esto sucede, una trabajadora so- cial se presenta en el domicilio del menor para constatar la denuncia y entregar un citatorio a los padres para que comparezcan en las instalaciones de dicha dependencia; asimismo, se les solicita que lleven al menor junto con ellos para que éste sea valorado física y psicológicamente por el equipo de salud (médico, psicólogo y trabajadora social). Si después de la exploración clínico-psicológica se comprueba el diagnóstico, se levanta un acta judicial y se determina si es pertinente el ingreso del niño al albergue para su tratamiento físico-psicológico; también se valora la conveniencia de tratar al o a los agresores.

En caso de que los padres o tutores del niño no se presenten en las instalaciones del DIF, o bien, cuando el diagnóstico es evidente, el Ministerio Público local y la trabajadora social, apoyados por elementos de la policía municipal, acuden al domicilio del menor para llevarlo a las instalaciones del DIF. El niño que es separado de su hogar permanece en el albergue hasta que las circunstancias de agresión desaparezcan o, por lo menos, hasta que las condiciones ambientales en el

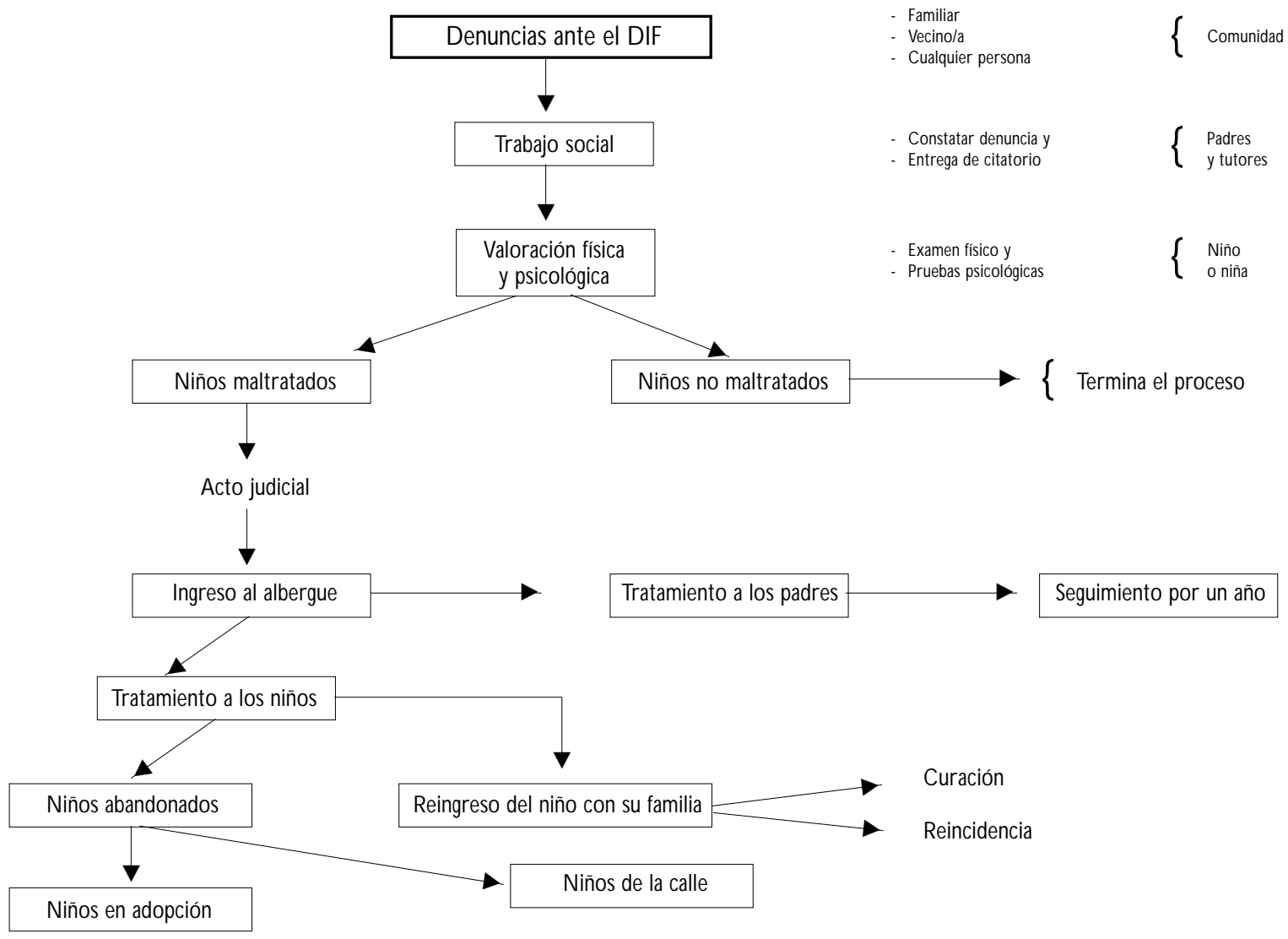

Figura 1. Ruta crítica para la detección y el tratamiento del niño maltratado. Sistema para el Desarrollo Integral de la Familia. México, 1999 
núcleo familiar mejoren mediante el tratamiento al o a los agresores. En muchos casos los niños son abandonados en el albergue; los padres cambian de domicilio y no se vuelve a saber de ellos. Las trabajadoras sociales tratan de localizar por todos los medios a los padres; cuando esto no es posible, pasado un lapso, buscan hogares adoptivos para el niño. En otros casos, cuando los padres cooperan y aceptan el tratamiento familiar, y cuando se corrobora que el ambiente familiar ha cambiado, se reintegra el niño a su hogar; posteriormente la trabajadora social realiza visitas periódicas para comprobar el cambio de actitudes y que el ambiente familiar haya mejorado.

\section{Conclusiones}

El presente ensayo tiene como propósito fundamental auxiliar, tanto al médico general institucional, de consulta externa y de urgencias de nuestros hospitales, como al médico privado, en el diagnóstico del niño maltratado o con síndrome de Kempe, a partir de una serie de indicadores de tipo clínico y psicológico.

Aunque no se conoce con toda certeza cuál es la frecuencia real de maltrato en el país, así como las características del mismo en las diferentes zonas, el maltrato debe ser reconocido como una patología y no como algo inherente a nuestra cultura. El médico debe tener la capacidad de diagnosticar o sospechar maltrato en todo paciente en el que se detecte alguno de los factores de riesgo, acompañado de signos físicos o psicológicos de maltrato evidentes; cabe añadir que la capacidad del médico para ganarse la confianza del niño y de la madre es fundamental para poder apoyarlos o asesorarlos en esta patología.

La identificación de los factores de riesgo más frecuentes para la presencia del síndrome del niño maltratado permitirá plantear estrategias de prevención y control del padecimiento, en un intento por detener este fenómeno social multicausal.

Es de vital importancia conocer tanto las instancias como los procedimientos legales y oficiales relacionados con el niño maltratado, ya que ello permitirá al médico asesorar a su paciente con mayor conocimiento y sustento jurídico acerca de las opciones legales que tiene para protegerse del agresor. El esquema que utiliza el DIF orienta al médico respecto de la conducta a seguir si llega a sospechar y detectar un caso de maltrato; lo guía tanto para notificar el caso, como para diagnosticar el síndrome, para tratar al paciente y darle seguimiento para su rehabilitación.

Por último, cabe añadir que es necesario el establecimiento de una norma oficial para prevenir la violencia intrafamiliar -incluyendo el maltrato a los niños-, donde se observe la obligatoriedad de la notificación de los casos y se señalen los procedimientos técnicos de diagnóstico, prevención, tratamiento y seguimiento de niños con síndrome de Kempe.

\section{Referencias}

1. Feigelson-Chase N. Un niño ha sido golpeado. La violencia contra los niños: una tragedia moderna. México, D.F.: D iana, 1980:32.

2. Friedlander W A. D inámica de trabajo social. México, D.F.: Pax, 1981:375.

3. Sainz-Villanueva M. El perfil social de los padres responsables en casos de niños maltratados del D.F. (tesis). México, D.F.: Instituto $\mathrm{N}$ acional de Psiquiatría, 1985.

4. Kempe HC, Silverman NF, Steele BF, D roegemuller W, Silver HK. The battered-child syndrome. Jama 1962;18(1):105-113.

5. FontanaV. En defensa del niño maltratado. México, D.F.: Pax, 1979.

6. Loredo AA. Maltrato al menor. México, D.F.: N ueva Editorial Interamericana/McG raw-Hill, 1994:162

7. 0 sorio CA. El niño maltratado. 2a. edición. México, D.F.:Trillas, 1995.

8. Kempe RS, Kempe HC. N iños maltratados. 4a. edición. Madrid: Morata, 1985.

9. Kempe HC. Pediatric implications of the battered baby syndrome.Arch D is Child 1996;46(28):28-37.

10. Aguilar AM. Maltrato a menores. En: Memorias Segundo Congreso Interdisciplinario e Internacional. El maltrato a los niños y sus repercusiones educativas. México, D.F.: Ficomi, 1992;2:300-307.

11. Santana TR, Irigoyen CA, Herrera BE. Síndrome de Kempe: pautas de diagnóstico para el médico general y familiar. Rev Fac Med UN AM 1997; 40:187-190

12. Ministerio de Salud de Chile. 0 rientaciones técnicas y programáticas en maltrato infantil. Pub Sal Mental 1998;5.

13. Santana TR, Sánchez AR, Herrera BE. El maltrato infantil: un problema mundial. Salud Publica Mex 1998;40:58-65.

14. Escobedo Ech, Loaeza-Fuentes V, Gómez-N ajera RS, Díaz-A guirre MJ, et al.Abuso sexual en pediatría: factores epidemiológicos. Bol Med Hosp Infant 1995;52(9):528-533. 